\title{
An advanced universal circulatory assist device for left and right ventricular support: First report of an acute in vivo implant
}

\author{
Takuma Miyamoto, MD, PhD, ${ }^{a}$ Yuichiro Kado, MD, PhD, ${ }^{a}$ David J. Horvath, MSME, \\ Barry D. Kuban, BS, ${ }^{\mathrm{a}, \mathrm{b}}$ Shiva Sale, MD, ${ }^{\mathrm{c}}$ Kiyotaka Fukamachi, MD, PhD, ${ }^{\mathrm{a}}$ and \\ Jamshid H. Karimov, MD, $\mathrm{PhD}^{\mathrm{a}}$
}

\begin{abstract}
Background: The Advanced ventricular assist device (Advanced VAD) is designed as a universal pump intended to prevent backflow in the event of pump stoppage, to maintain physiological pulse pressure, and to be used as both a left and right VAD. The purpose of this study was to evaluate the performance of the Advanced VAD as both a left and right VAD in an acute in vivo study in calves.

Methods: The Advanced VAD was implanted through a median sternotomy in 5 healthy calves (weight, 71.4-91.2 kg) as a left $\operatorname{VAD}(n=3)$ or a right $\operatorname{VAD}(n=2)$. After implantation, hemodynamic parameters, including general performance and pump stoppage, were evaluated.

Results: The Advanced VAD was successfully implanted as a left and right VAD without cardiopulmonary bypass. The speed range of the Advanced VAD was 2500 to $3500 \mathrm{rpm}$ as a left VAD and 2000 to $2500 \mathrm{rpm}$ as a right VAD. Up to $4.3 \mathrm{~L} / \mathrm{min}$ was achieved for both left and right VAD configurations. To demonstrate the automatic shut-off feature, the pump was stopped without clamping the outflow graft. The outflow graft was then clamped, which produced no significant changes in the arterial pressure waveform. The pulse pressures under the left VAD configuration were $38 \mathrm{~mm} \mathrm{Hg}, 17 \mathrm{~mm} \mathrm{Hg}, 14 \mathrm{~mm} \mathrm{Hg}$, and $16 \mathrm{~mm} \mathrm{Hg}$ at baseline, $2500 \mathrm{rpm}, 3000 \mathrm{rpm}$, and $3500 \mathrm{rpm}$, respectively.
\end{abstract}

Conclusions: This acute in vivo study demonstrated the pump performance, anatomical fitting as both left VAD and right VAD, and regurgitant flow shut-off feature of the Advanced VAD. (JTCVS Open 2020;3:140-8)

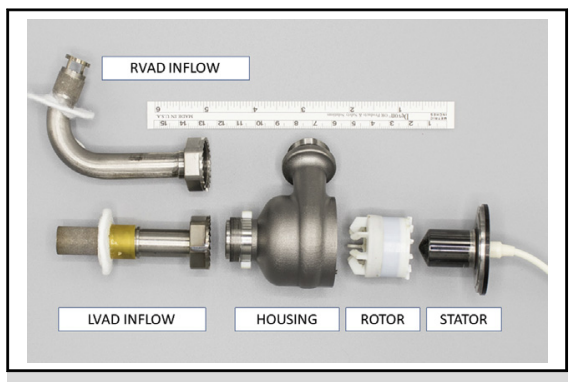

Advanced ventricular assist device components.

CENTRAL MESSAGE

An acute in vivo study was conducted to evaluate the performance of the new ventricular assist device with wide operational range, pulsatility augmentation, and regurgitant flow shut-off features.

\section{PERSPECTIVE}

To address the limitations of current ventricular assist therapy, such as loss of pulsatility, severe backflow during pump stoppage, and right ventricular failure, we developed the Advanced ventricular assist device. This in vivo acute study demonstrated the pump performance, anatomical fitting as both left and right heart support, and pulsatile flow and regurgitant flow shut-off feature of the pump.

See Commentaries on pages 149,150 , and 152
From the ${ }^{\mathrm{a}}$ Department of Biomedical Engineering, Lerner Research Institute, ${ }^{\mathrm{b}} \mathrm{Med}$ ical Device Solutions, and ${ }^{\mathrm{c}}$ Department of Cardiothoracic Anesthesiology, Cleveland Clinic, Cleveland; and ${ }^{\mathrm{d}} \mathrm{R} 1$ Engineering, Euclid, Ohio.

The Advanced Ventricular Assist Device Program is supported by federal funding from the National Heart, Lung, and Blood Institute under grant 5R21HL133871 (to K.F.).

Received for publication May 29, 2020; revisions received May 29, 2020; accepted for publication June 16, 2020; available ahead of print July 22, 2020.
Address for reprints: Jamshid H. Karimov, MD, PhD, Department of Biomedical Engineering, Cleveland Clinic, 9500 Euclid Ave/ND20, Cleveland, OH 44195 (E-mail: karimoj@ccf.org).

2666-2736

Copyright (C) 2020 The Authors. Published by Elsevier Inc. on behalf of The American Association for Thoracic Surgery. This is an open access article under the CC BY-NCND license (http://creativecommons.org/licenses/by-nc-nd/4.0/).

https://doi.org/10.1016/j.xjon.2020.06.006 


\section{Abbreviations and Acronyms \\ AoP $=$ aortic pressure \\ $\mathrm{CF}=$ continuous flow \\ $\mathrm{dP} \quad=$ pump differential pressure \\ LVAD $=$ left ventricular assist device \\ PVR = pulmonary vascular resistance \\ $\mathrm{RVAD}=$ right ventricular assist device \\ SVR = systemic vascular resistance \\ $\mathrm{VAD}=$ ventricular assist device}

Video clip is available online.

Ventricular assist devices (VADs) have been widely used to treat end-stage heart failure. According to the eighth annual report from the Interagency Registry for Mechanically Assisted Circulatory Support, 18,591 Food and Drug Administration-approved VADs were implanted between June 2006 and December 2016 at 185 participating hospitals, and more than $90 \%$ were continuous-flow (CF) left ventricular assist devices (LVADs). ${ }^{1}$ The rate of patient enrollment has grown to exceed 2500 implants per year for durable mechanical circulatory assist devices such as LVADs and total artificial hearts. Device strategy is changing such that the number of LVADs implanted as destination therapy has continued to grow. However, as support duration increases, clinical limitations and specific complications of CF-LVADs have become apparent. These include a loss of arterial pulsatility, ${ }^{2-4}$ development of aortic regurgitation, ${ }^{5}$ pump thrombosis or hemolysis, ${ }^{6,7}$ severe backflow during pump stoppage, difficulty evaluating native heart function and weanability, ${ }^{8,9}$ and acute or late right heart failure. ${ }^{10,11}$

In addition, despite major breakthroughs with CF mechanical circulatory system technology in recent years, major efforts were made to deliver LVADs to clinical practice. However, there are still no durable right ventricular assist devices (RVADs) available to address right heart failure. Existing VADs are not specifically designed for right ventricular support, which is limited by LVAD operational range. ${ }^{12}$

The Advanced VAD, which is under development at our institution and is intended to be used as a universal device (suitable for left and right heart assist or biventricular support), is designed to address those limitations by introducing an automatic and dynamic pump performance regulation property also found in our CF total artificial heart. ${ }^{13}$ This pump's novel feature is that the rotor can move axially to regulate the impeller discharge aperture size, depending on the pressure difference between the inlet and outlet and pump speed. This feature makes the pump more sensitive to pressure differences in the pump, allowing the performance to dynamically change during the cardiac cycle. We have shown previously in vitro that: (1) this pump can increase pulse pressure compared with existing CF-LVADs; (2) the regurgitant flow shut-off feature prevents backflow during pump stoppage, enabling a more accurate evaluation of native heart function; and (3) the wide performance range allows the device to be used as an LVAD, an RVAD, and a biventricular assist device with 2 pumps. ${ }^{14}$ The purpose of the present study was to evaluate the performance and the specific properties of the Advanced VAD in an acute animal model under both LVAD and RVAD configurations.

\section{METHODS \\ Device Description}

The cross-section of the pump is shown in Figure $1, A$. This is a magnetically and hydrodynamically levitated centrifugal pump, and the performance range is 2000 to $3500 \mathrm{rpm}$ and up to $10 \mathrm{~L} / \mathrm{min}$. The pump is controlled with a custom-built control unit consisting of a microcontroller-based motor drive circuit with a built-in power supply and rotary dial speed selector. The pump components, including titanium inflow cannulae are shown in Figure 1, B. The pump length is $57 \mathrm{~mm}$ and pump diameter is $44 \mathrm{~mm}$, with a total pump displacement volume of $70 \mathrm{~mL}$ and a priming volume of $15 \mathrm{~mL}$. The housing components of the current prototype are made of titanium, and the rotor is made of plastic with reduced clearance modification from previous study results. ${ }^{15}$ The LVAD inflow cannula is open ended and straight and the length is $69 \mathrm{~mm}$ and $14 \mathrm{~mm}$ in diameter. The RVAD inflow cannula is open-ended with 2 side holes and angled, and the length is $125 \mathrm{~mm}$ and $14 \mathrm{~mm}$ in diameter.

The novel design aspect of the pump is that the motor magnet in the rotor is shorter than the motor stator winding (Figure 1, A), so that the rotor can move axially to some extent ${ }^{13}$ (US Patent: US 2018/0311426 A1). The axial spacing between the impeller and housing (aperture) varies because of this axial movement at startup and during operation (Figure 1, A), depending on the pressure difference in the pump (pump differential pressure $[\mathrm{dP}]$ ) and rotational speed and range of the aperture opening (Video 1). A magnetic force acts to close the aperture, and the hydraulic forces during rotor rotation act to open it. This small but dynamic axial movement of the rotor changes the performance in response to the cardiac cycle. During diastole (pump dP is high), the rotor moves toward the aperture and decreases the flow. During systole (pump $\mathrm{dP}$ is low), the rotor moves against the aperture and increases the flow. In this way, the Advanced VAD is expected to provide increased pulsatility of the pump flow and pressure (pulse augmentation). When the rotor stops (power off), the hydraulic forces diminish, and the magnetic force immediately moves the rotor to close the aperture and shut down the flow (regurgitant flow shut-off). If the pump runs in a lower-pressure environment such as the pulmonary circulation, the aperture opening is automatically reduced, and the entire pump performance is decreased to provide adequate flow to the pulmonary circulation (RVAD use).

\section{In Vitro Study}

Bench testing with the Advanced VAD was performed on the static mock loop to obtain pressure-flow curves at various pump speeds before each in vivo experiment. A glycerin/water mixture (specific gravity, 1.060) was used as the working fluid. Vascular resistances were modeled with manual valves, arterial compliances were modeled with closed pneumatic reservoirs, and the pump inlets were fed from open reservoirs. In each test, data were recorded across the full range of operation (rotational 

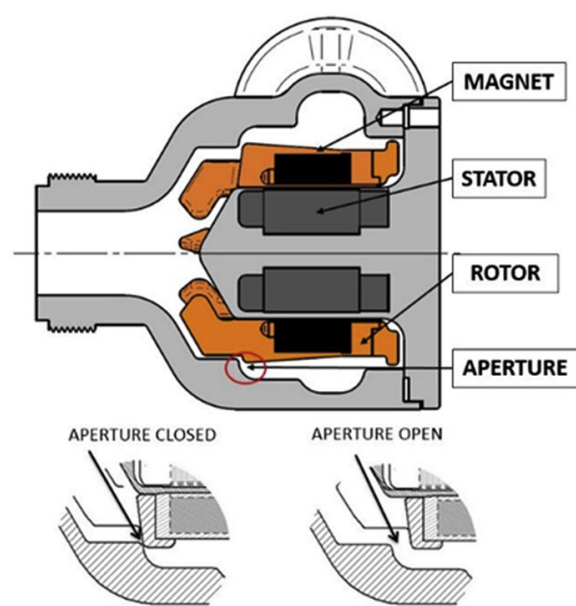

A

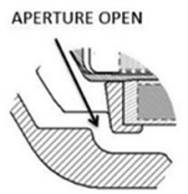

FIGURE 1. A, Cross-section illustration of the Advanced ventricular assist device: the pump is made of a housing, a stator, and a rotor. The rotor can move axially depending on pressure difference in the pump, and can change the space at the aperture. B, The photo of the Advanced ventricular assist device components: the straight inflow cannula was used for left ventricular support, and the angled inflow cannula was used for right ventricular support. $R V A D$, Right ventricular assist device; $L V A D$, left ventricular assist device.

speed of 2000-3500 rpm, aortic pressure of 20-120 mm Hg). The pressure rise was calculated as the outlet minus inlet pressures.

\section{Surgical Approach}

The study was approved by the Cleveland Clinic's Institutional Animal Care and Use Committee (\#2016-1596). A total of 5 acute in vivo studies were performed using male Jersey calves (mean body weight $=83.2 \mathrm{~kg}$, range $=72.4-91.2 \mathrm{~kg}$ ).

Under general anesthesia, arterial pressure (AoP) and central venous pressure monitoring were placed in the carotid and jugular veins, respectively. A median sternotomy was performed, and heparin $(300 \mathrm{U} / \mathrm{kg})$ was administered for systemic heparinization. No cardiopulmonary support was used. The outflow graft was end-to-side anastomosed to the ascending

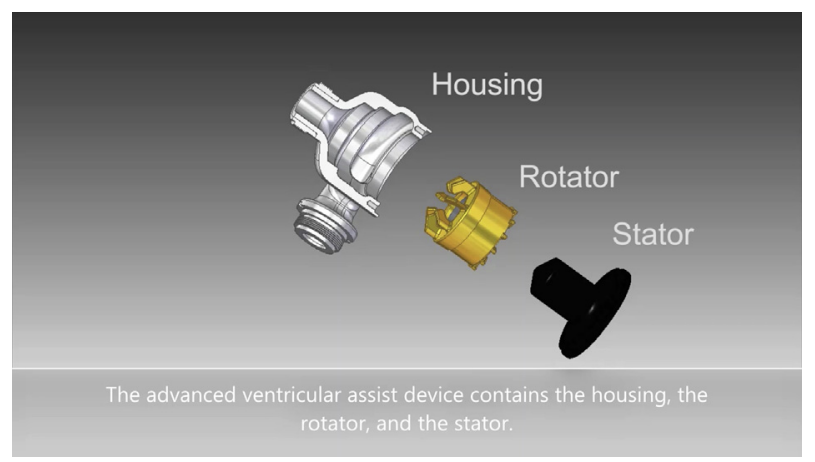

VIDEO 1. The video shows the pump components and novel features. The pump consists of the housing, the rotor, and the stator. The axial spacing between the impeller and housing (aperture) varies because the rotor moves axially at startup and during operation, depending on the pressure difference in the pump and rotational speed. In this way, the pump is expected to provide increased pulsatility of the pump flow and pressure (pulse augmentation). When the rotor stops (power off), the hydraulic forces diminish, and the magnetic force immediately moves the rotor to close the aperture and shut down the flow (regurgitant flow shut-off). Video available at: https://www.jtcvs.org/article/S2666-2736(20)30055-3/fulltext. aorta (RVAD: pulmonary trunk). The pressure line was placed in the left ventricle through the left atrial appendage. Purse string sutures were placed on the apex (RVAD: right ventricular free wall) and cored with a coring tool, then an inlet cannula was inserted and secured. The outflow conduit was connected to the pump and de-aired, and the pump was started. Upon testing completion, the animal was fully heparinized (500 U/kg), the pump was stopped, and the study was terminated.

\section{Hemodynamic Conditions}

The measured hemodynamic and pump performance data included the following: (1) heart rate; (2) central venous pressure (fluid-filled catheter at the jugular vein); (3) AoP (fluid-filled catheter in the peripheral artery and on the outlet graft); (4) pulmonary artery pressure (fluid-filled catheter to the outflow graft, only for the RVAD study); (5) left ventricular pressure or pump inlet pressure (fluid-filled catheter); (6) pump flow (inline flow probe [ME-13 PXN Inline Flow Sensor; Transonic, Ithaca, NY], in the outlet conduit); (7) motor current (analog output from the control module); and (8) motor speed (analog output from the control module). In each condition, these hemodynamics were recorded about 5 seconds after the steady state was reached.

Hemodynamic and pump performance data were collected in the following manner:

(1) Speed range test. Data were obtained at baseline (without pump support) and at $2000 \mathrm{rpm}, 2500 \mathrm{rpm}, 3000 \mathrm{rpm}$, and $3500 \mathrm{rpm}$.

(2) Pump stoppage test. The pump was stopped to evaluate the shut-off feature. The pump was run at $3000 \mathrm{rpm}$ for the LVAD configuration and at 2000 or $2500 \mathrm{rpm}$ for the RVAD configuration. The pump was stopped for $\geq 5$ seconds using the controller, and then the outflow conduit was clamped to verify the effectiveness of the automatic shut-off feature. The hemodynamic parameters and regurgitation flow were recorded a total of 10 times.

(3) Inlet cannula malposition suction control test. While in fixed-speed mode, the inlet cannula was rotated toward the interventricular septum, lateral wall, or aortic valve until flow obstruction occurred.

(4) High systemic vascular resistance (SVR) or pulmonary vascular resistance (PVR) test. High SVR or PVR was induced by clamping the outflow graft or aorta (RVAD: the pulmonary artery) partially, approximating $50 \%, 75 \%$, and $90 \%$ obstruction. 
(5) Low SVR or PVR test. A state of low SVR or PVR (LVAD: $<800$ \pm 50 dyne $\cdot \mathrm{sec} \cdot \mathrm{cm}^{-5}$, AoP: 50-70 mm Hg, RVAD: < $100 \pm 50$ dyne $\cdot \mathrm{sec} \cdot \mathrm{cm}^{-5}$, mean pulmonary artery pressure: $5-10 \mathrm{~mm} \mathrm{Hg}$ ) was induced by isoflurane (up to $5 \%$ ).

Acute hemodynamic and pump performance parameters were digitized in real time at a sampling rate of $200 \mathrm{~Hz}$ with a PowerLab (ADInstruments Inc, Colorado Springs, Colo) data acquisition system. The data were analyzed using a custom-made visual basic program in Excel software (Microsoft Corporation, Redmond, Wash). The pulse pressure was calculated as (maximum AoP - minimum AoP).

\section{RESULTS}

\section{Overall Outcome}

The Advanced VAD was successfully implanted as an $\operatorname{LVAD}(\mathrm{n}=3)$ and as an RVAD $(\mathrm{n}=2)$ without cardiopulmonary bypass support (Figure 2, A: LVAD; Figure 2, $B$ : RVAD). There were no complications during the procedure, and anatomical fitting was acceptable. The speed range of the Advanced VAD was 2000 to $3500 \mathrm{rpm}$ as an LVAD and 2000 to $3000 \mathrm{rpm}$ as an RVAD, and up to $4.3 \mathrm{~L} / \mathrm{min}$ (1.1-4.3 L/min) was achieved for both configurations. The pump performance was compared with a bench test (Figure 3, A). The pump performance was observed to be lower in vivo at high speed (3000 or $3500 \mathrm{rpm}$ ) due to some degree of inlet suction; the overall pump performance was shown to be consistent throughout the experiments with the values observed during preoperative in vitro device testing. Figure 3, $B$, shows representative dynamic pressure flow loops of the Advanced VAD during cardiac cycles implanted as an LVAD. The widest pressure flow loop was observed at $2500 \mathrm{rpm}$, indicating the greatest pulsatility. The smallest loops were observed at $3000 \mathrm{rpm}$ and $3500 \mathrm{rpm}$.

The charts from both the LVAD and RVAD studies from selected experiments are shown in Figure 4. In Figure 4, A (LVAD configuration), as pump speed increased from 2000 to $2500 \mathrm{rpm}$, the pump flow increased from $1.5 \mathrm{~L} / \mathrm{min}$ to $2.9 \mathrm{~L} / \mathrm{min}$, and the average inlet pressure decreased from $30 \mathrm{~mm} \mathrm{Hg}$ to $23 \mathrm{~mm} \mathrm{Hg}$. However, at $3000 \mathrm{rpm}$ and 3500 $\mathrm{rpm}$, the average inlet pressure went down to $3 \mathrm{~mm} \mathrm{Hg}$ and $-28 \mathrm{~mm} \mathrm{Hg}$, respectively, and the pump flow did not increase $(3.0 \mathrm{~L} / \mathrm{min}, 3.0 \mathrm{~L} / \mathrm{min}$ respectively) due to inlet suction. During the inlet cannula malposition test, we successfully produced the suction condition; pump flow decreased when the inlet attached to the ventricular wall (1.3-3.0 L/min). The pump was sensitive to high and low SVR, as the pump flow significantly decreased as SVR increased and caused the suction with low SVR. In the RVAD configuration (Figure 4, $B$ ), as pump speed increased from 2000 to 2500 , the pump flow increased from 3.2 to $4.3 \mathrm{~L} / \mathrm{min}$, but the inlet pressure dropped to negative, meaning inlet suction, so we decided to use the data with $2000 \mathrm{rpm}$. RVAD was more stable in the cannula malposition than LVAD. RVAD is also sensitive to high PVR, as the pump flow significantly decreased as PVR increased.

\section{Regurgitant Flow Prevention Testing}

The regurgitant flow (backflow) feature of the Advanced VAD was successfully evaluated in this series of studies for both the LVAD and RVAD configurations. The representative waveforms observed for the following modes were recorded: on-pump; off-pump without clamping; and offpump with clamping (Figure 5, A). When the pump was stopped, there was trivial regurgitant flow through the pump. Therefore, there were no changes in the arterial pressure waveform observed when the outflow graft was clamped, suggesting that the pump-off test could be fully achievable without clamping the outflow conduit. Regurgitant flow after stopping the pump for each pump configuration is shown in Figure 5, B. When the pump was stopped, the mean regurgitant flow was $-0.3 \pm 0.0 \mathrm{~L} / \mathrm{min}$ for the LVAD configuration and $-0.7 \pm 0.3 \mathrm{~L} / \mathrm{min}$ for the RVAD configuration. The regurgitant flow tended to be lower for the LVAD configuration than for the RVAD configuration, but was less than $1.0 \mathrm{~L} / \mathrm{min}$ in all experiments.

\section{Pulse Augmentation Testing}

Figure 6, A, shows the representative waveforms for AoP, pump outlet pressure at the outflow conduit, and pump flow at $2500 \mathrm{rpm}$ with the Advanced VAD support as an LVAD. The pump support demonstrated an effective operational pressure pulsatility at the pump outlet and flow pulsatility. As shown in Figure 6, A, the aperture of the pump closes at the bottom of the flow curve and opens at the peak of the flow curve. Despite the pressure, pulsatility appeared somewhat absorbed at the outflow conduit. This was one of the several most effectively achieved pulse augmentations during the in vivo study, and at this point, the pulse pressure of AoP was $15 \mathrm{~mm} \mathrm{Hg}$, the pulse pressure of pump outlet was $63 \mathrm{~mm} \mathrm{Hg}$, and the flow range was from $0.2 \mathrm{~L} / \mathrm{min}$ to $6.0 \mathrm{~L} / \mathrm{min}$ Figure $6, B$, shows the mean pressure and flow pulsatility for the LVAD experiments. The mean pulse pressures for the LVAD configuration $(\mathrm{n}=3)$ were $38 \mathrm{~mm} \mathrm{Hg}$ (range: $32-45 \mathrm{~mm} \mathrm{Hg}$ ), $17 \mathrm{~mm} \mathrm{Hg}$ (range: 15 $19 \mathrm{~mm} \mathrm{Hg}$ ), $14 \mathrm{~mm} \mathrm{Hg}$ (range: 6-19 $\mathrm{mm} \mathrm{Hg}$ ), and $16 \mathrm{~mm}$ $\mathrm{Hg}$ (range: $8-18 \mathrm{~mm} \mathrm{Hg}$ ) at baseline, $2500 \mathrm{rpm}$, $3000 \mathrm{rpm}$, and $3500 \mathrm{rpm}$, respectively. The mean flow pulsatilities (peak flow - minimum flow) were $5.0 \mathrm{~L} / \mathrm{min}$, $3.9 \mathrm{~L} / \mathrm{min}$, and $4.2 \mathrm{~L} / \mathrm{min}$ at $2500 \mathrm{rpm}, 3000 \mathrm{rpm}$, and $3500 \mathrm{rpm}$, respectively.

\section{DISCUSSION}

The device performance was successfully tested in this series of in vivo studies. To our knowledge, this is the first report of the in vivo use of a VAD that is designed to be used as LVAD and RVAD support. The Advanced VAD is also the first device designed to address the complications related to the presence of regurgitant flow by enabling a shut-off feature. The device 

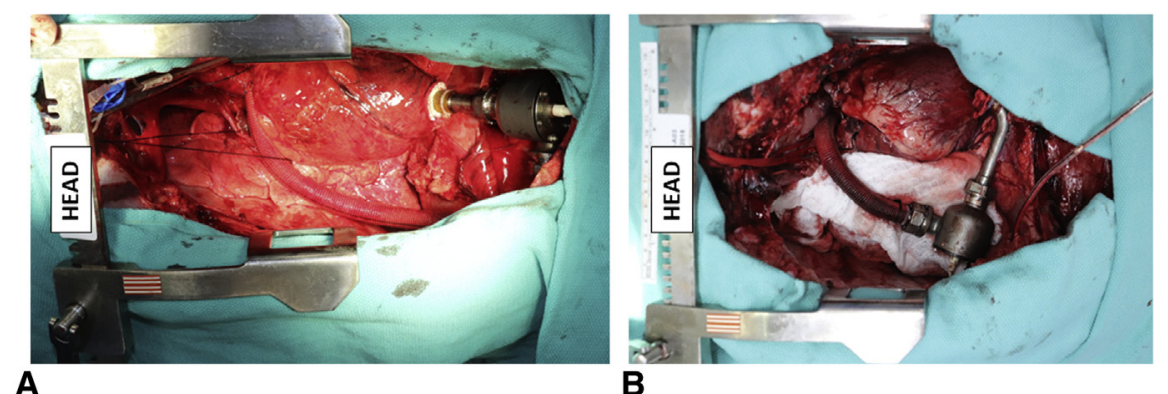

FIGURE 2. Intraoperative images of pump implantation. A, Left ventricular assist device: the inflow cannula was inserted into the left ventricular apex and the outflow graft was anastomosed to the ascending aorta. The pump sat on the diaphragm. B, Right ventricular assist device: the inflow cannula was inserted into the right ventricular diaphragmatic surface and the outflow graft was anastomosed to the pulmonary trunk. The pump sat in the right pleural cavity.

is also designed to provide pulse augmentation during circulatory support. In this acute in vivo study, we demonstrated that the Advanced VAD implantation was technically feasible, did not require cardiopulmonary bypass support, and showed intended hemodynamic performance at an acceptable range as a universal pump. The device is intended to be used as a single LVAD and/or RVAD and can provide adequate blood flow for
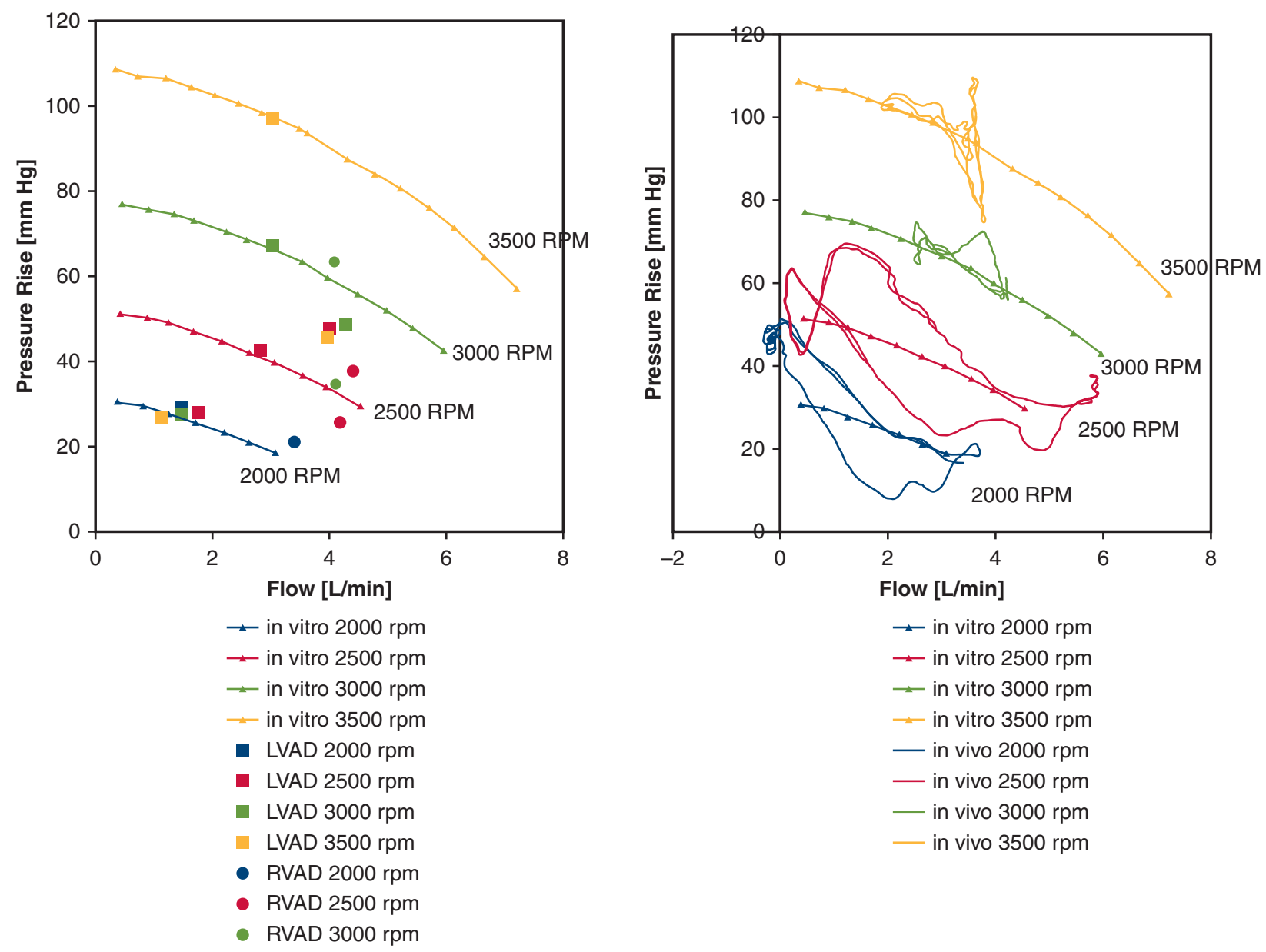

A

B

FIGURE 3. A, The pressure flow curves of the Advanced ventricular assist device from an in vitro study and the pump performance during an in vivo study: pressure rise was calculated as the outlet pressure minus inlet pressure (left ventricular pressure); the flow was measured at the outflow conduit. B, The representative pressure-flow loop during in vivo study: in vitro results are also shown as pressure flow curves. $L V A D$, Left ventricular assist device; $R V A D$, right ventricular assist device. 


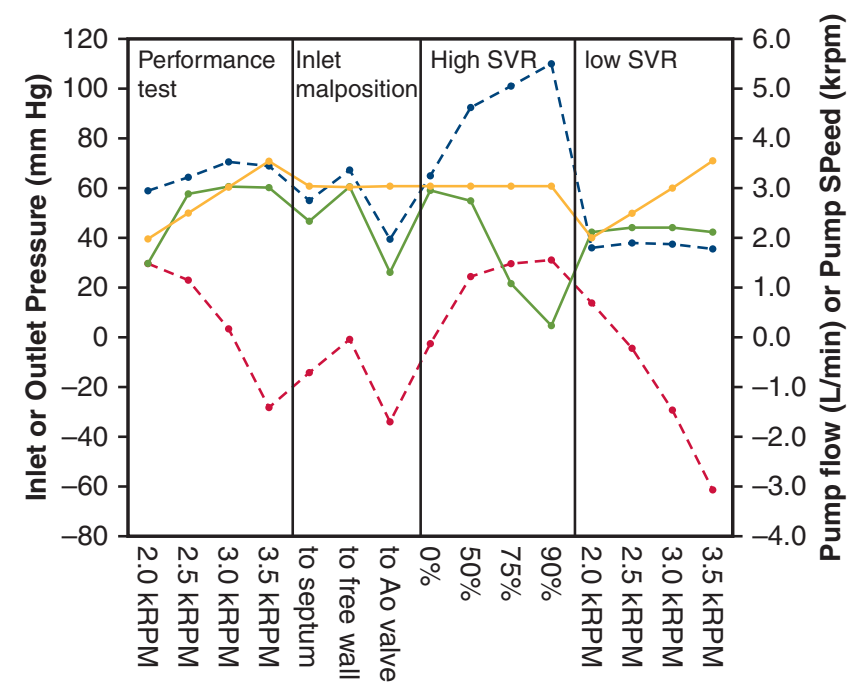

- -. Outlet Pressure -... Inlet Pressure

A

$\rightarrow$ Pump Flow $\rightarrow$ Speed

FIGURE 4. Representative chart of inlet pressure, outlet pressure, pump flow, and pump speed from left ventricular assist device configuration (A), and right ventricular assist device configuration (B) during in vivo experiments. SVR, Systemic vascular resistance; $P V R$, pulmonary vascular resistance. either condition. In addition, the effectiveness of the regurgitant flow shut-off feature and the potential pulse augmentation feature of the pump has been successfully tested in this series.

We observed that the regurgitant flow shut-off feature of this pump was reproducible in vivo. This specific feature enables evaluation of the native ventricular function without clamping or occluding the outflow graft and makes explantation of the pump much easier.

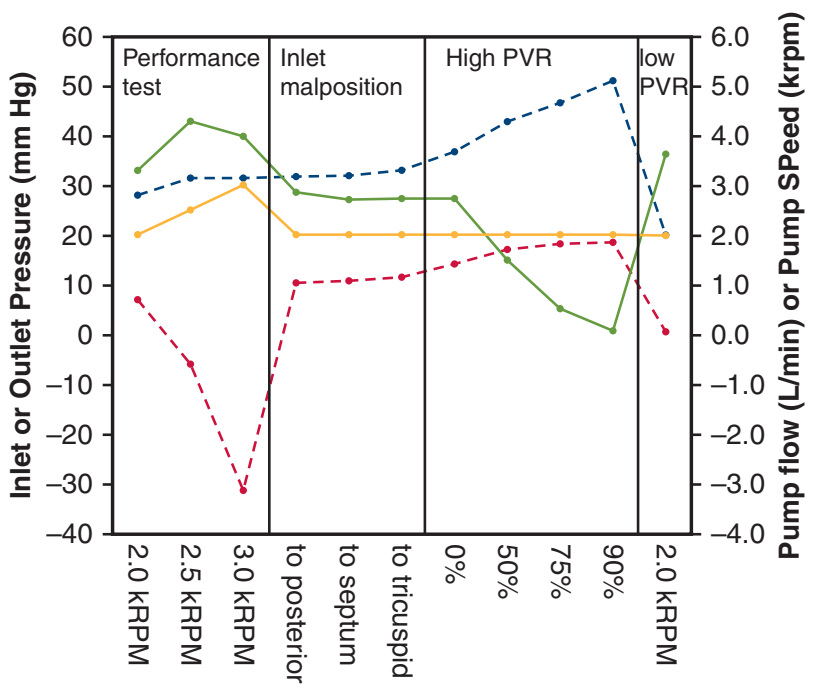

-... Outlet Pressure -... Inlet Pressure

$\rightarrow$ Pump Flow $\rightarrow$ Speed
B

Several groups reported their outcomes based on various clinical protocols and approaches for weaning the pump. Knierim and colleagues ${ }^{16}$ reported their weaning protocol and success rate among 424 patients implanted with LVADs, and reported 14 patients who underwent LVAD explantation. These authors demonstrated three steps of their weaning evaluation protocol, which included screening, echocardiography at "baseline," "zero net flow," and "pump stop conditions," as

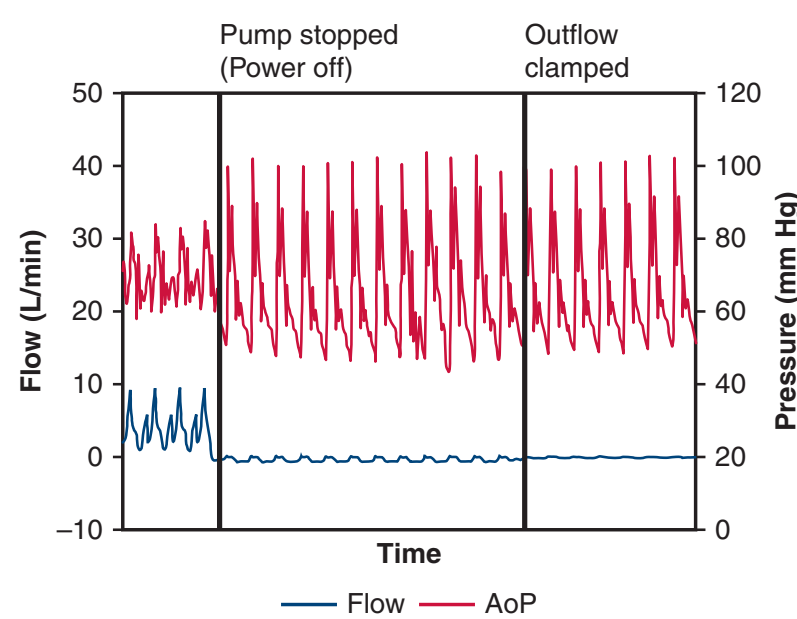

A

FIGURE 5. A, The representative waveforms for aortic pressure and pump flow when the pump was stopped (power off) without outflow graft clamping, and when the outflow graft was clamped. When the pump was stopped, the pump flow immediately fell to almost zero without regurgitant flow, and the flow waveform showed almost no change after the outflow graft was clamped, demonstrating the regurgitant shut-off feature of the pump. B, Mean regurgitant flow for the LVAD (red) and RVAD (blue): Mean regurgitant flow was $-0.3 \pm 0.0 \mathrm{~L} / \mathrm{min}$ for the LVAD configuration and $-0.7 \pm 0.3 \mathrm{~L} / \mathrm{min}$ for the RVAD configuration. AoP, Aortic pressure; $L V A D$, left ventricular assist device; $R V A D$, right ventricular assist device. 


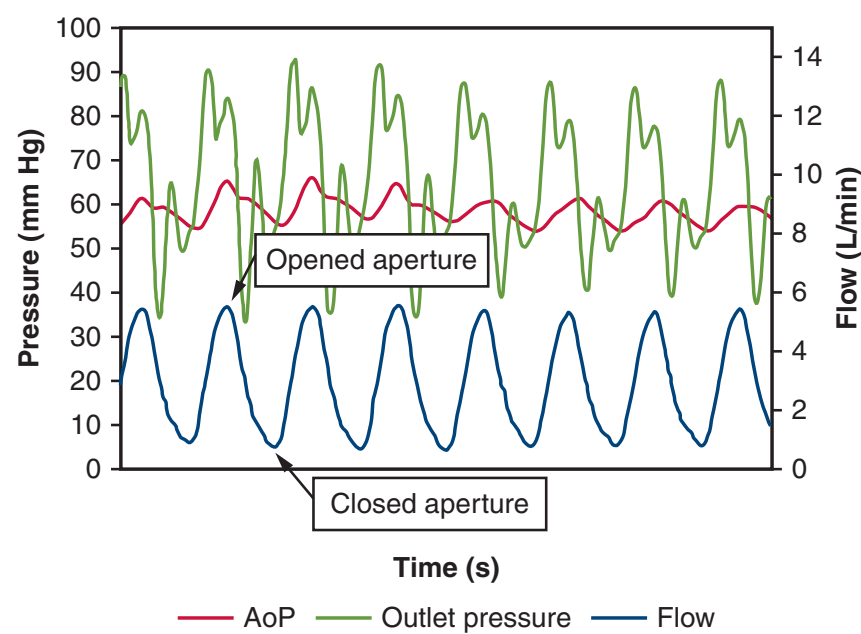

A

FIGURE 6. A, Representative waveform of AoP, pump outlet pressure, and pump flow at $2500 \mathrm{rpm}$ as an LVAD: the aperture of the pump opened during systole and closed during diastole as indicated in figure. B, Pressure and flow pulsatility in the LVAD configuration. The range of the bar shows the pressure and flow pulsatility; the middle line inside the bar show the mean AoP or pump flow. AoP, Aortic pressure.

well as right heart catheterization with graft occlusion and pump stoppage. No complications during the evaluation were reported, but as discussed, the "zero net flow" condition could not be considered accurate, as the "pump stop condition" causes massive regurgitant flow and acute myocardial stress, ${ }^{17}$ and the balloon occlusion of the outflow graft creates the potential for complications, such as bleeding and thromboembolism. ${ }^{18}$ Our previous in vitro studies to evaluate the HeartMate II (Abbott Inc, Chicago, Ill) and HVAD (HeartWare Inc, Framingham, Mass) performance at very low pump speeds showed that even at low speed, those pumps have a significant contribution to mean AoP and total flow, especially under heart failure conditions. ${ }^{8,9}$ The Advanced VAD would allow pump evaluation without regurgitation during echocardiographic assessment and thus would assist with the evaluation and assessment of weaning strategies in heart failure patients implanted with this VAD.

The Advanced VAD is designed to perform universally as both an LVAD and an RVAD. In the RVAD configuration with low rotational speed, the rotor moves to close the aperture and reduce pump performance, so this pump has a wider performance range than other existing devices. The typical operational range required for the CF-RVAD is to generate pump flows of 2 to $6 \mathrm{~L} / \mathrm{min}$ while raising the pump outlet pressure by 20 to $50 \mathrm{~mm} \mathrm{Hg}$ from that at the pump inlet port. ${ }^{19}$ As reported in the in vitro study, the Advanced VAD can generate up to $6 \mathrm{~L} / \mathrm{min}$ while raising the pump outlet pressure 20 to $50 \mathrm{~mm} \mathrm{Hg}$ at $2500 \mathrm{rpm}$. In the in vivo study, the mean pump flow was

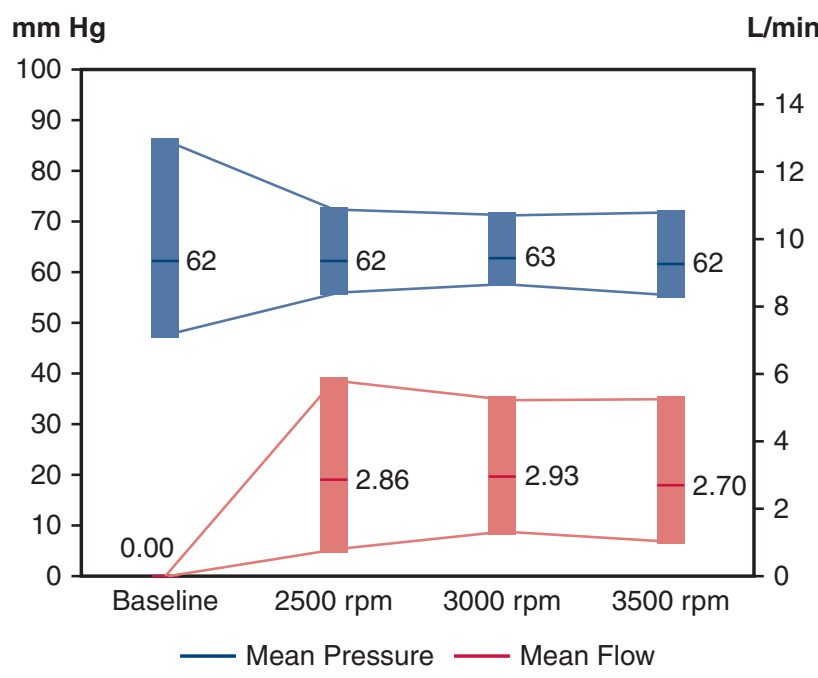

$3.8 \mathrm{~L} / \mathrm{min}$ and $4.3 \mathrm{~L} / \mathrm{min}$ at $2000 \mathrm{rpm}$ and $2500 \mathrm{rpm}$, respectively, suggesting that a rotational speed between 2000 and $2500 \mathrm{rpm}$ should be appropriate for right ventricular support, and 3000 and $3500 \mathrm{rpm}$ for left ventricular support. From the HVAD pressure flow curve, which was recorded in our institution, the pump speed was $1800 \mathrm{rpm}$ to get similar flow at RVAD pressure rise, which is less than the recommended clinical speed range (2400$3200 \mathrm{rpm}$ ).

HVAD is currently the most frequently used as an RVAD (off-label use). To fit LVAD performance to right heart support, many physicians use a relatively long outflow graft or narrow the outflow graft to increase the afterload, ${ }^{20,21}$ and the pump inflow is inserted into the right atrium to prevent suction. ${ }^{21}$ Restricting the outflow graft changes the flow distribution in the graft and has a potential risk of thrombus formation. The restriction cannot be adjusted after implantation, while the Advanced VAD adjusts its performance automatically. ${ }^{22}$ Shah and colleagues ${ }^{23}$ reported a multicenter study of 46 patients who received a durable biventricular VAD (HVAD); suspected RVAD thrombosis developed in 17 patients $(37 \%)$. There was a trend toward lower rates of suspected RVAD thrombosis with a right ventricle cannulation $(26 \%)$ compared with a right atrial cannulation $(48 \%, P=.13)$ implant. Patients with an RVAD thrombosis had a lower pump operational speed (2300 rpm) than patients without RVAD thrombosis $(2450 \mathrm{rpm}, P=.22)$. Although studies have shown acceptable results, using the HVAD as an RVAD may not be optimal, and a pump suitable for the hemodynamics of the pulmonary circulation still needs to be developed. ${ }^{24}$ 

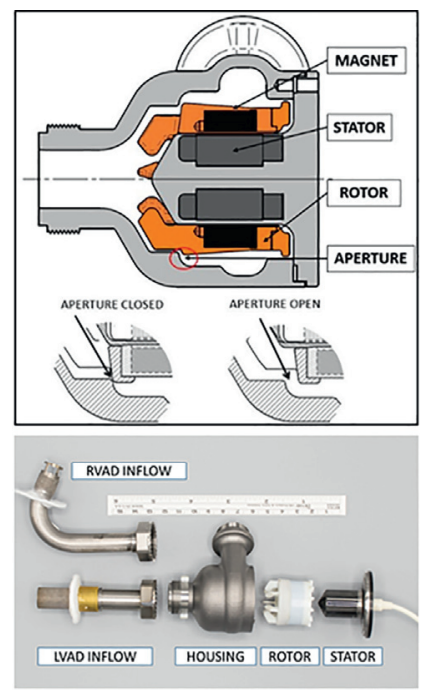

The advanced ventricular assist device has novel features. 1. works as left and right ventricular assist device. 2. prevents regurgitant flow.
3. augments pulsatile flow

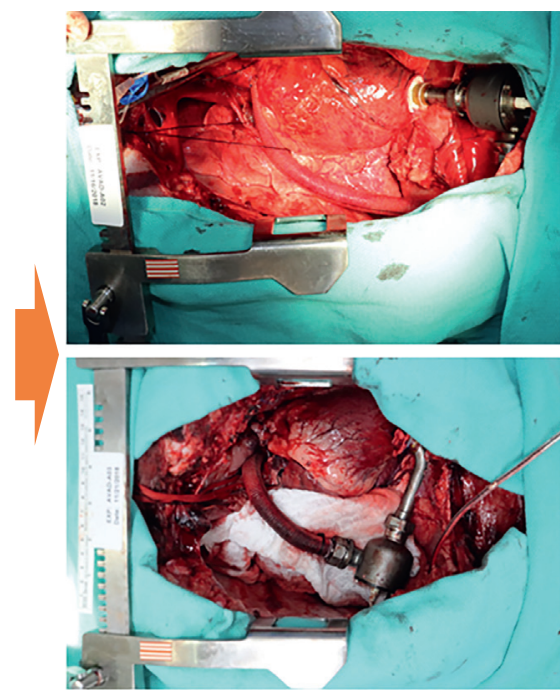

The pump was implanted to 5 healthy calves $(3$ to left ventricule and 2 to right ventricle), and observed hemodynamics and novel features in acute in vivo study.
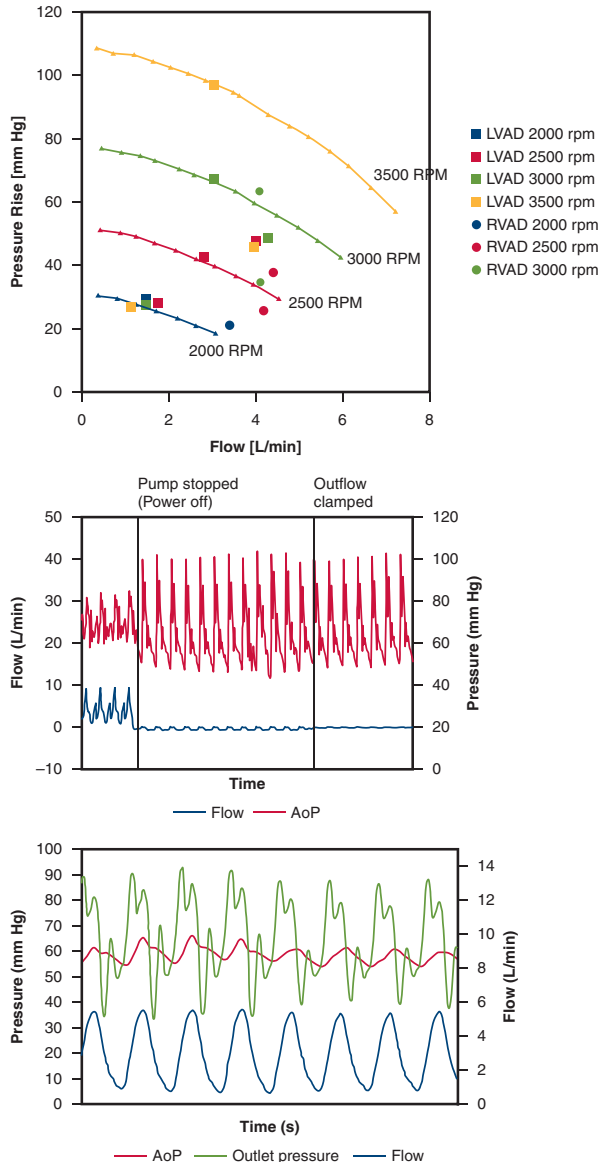

— AoP — Outlet pressure — Flow

The pump worked as both left and right ventricular assist device prevented regurgotant flow when the pump was stopped, and
preserved pulsatility.

FIGURE 7. The Advanced ventricular assist device is a universal pump designed to be used as a left, right, and biventricular ventricular assist device and also has features to preserve arterial pulsatility and prevent regurgitant flow while the pump is off. We report here the first acute animal study of the universal Advanced ventricular assist device implanted through a median sternotomy in calves $(\mathrm{n}=5)$. The pump kept the hemodynamics stable as both left $(\mathrm{n}=3)$ and right $(\mathrm{n}=2)$ heart support, had a regurgitant flow shut-off feature and offered potential pulse augmentation. $L V A D$, Left ventricular assist device; $R V A D$, right ventricular assist device; $A o P$, aortic pressure.

There are some limitations to this study. First, healthy animals were used in these experiments. The ventricular cavity of the healthy calves appeared to be of smaller dimensions, and in the end-systolic phase, the ventricles were fully ejected. The pump should be more resistant to suction events than existing pumps, because when suctions occur, the pump aperture automatically closes and lowers the flow, reducing the suction on the tissue. Second, the pump is designed to be fully implanted in the chest and intended to fit in the pericardial space. The long inflow cannula is designed for in vivo studies with normally hypertrophic hearts in calves and enables us to adjust the pump position easily during acute animal study. ${ }^{25}$ Third, the pump showed lower pulsatility augmentation than in the in vitro study. Some factors could affect the results, such as the length of the outflow conduit, compliance difference, or preload sensitivity of the native heart. To enhance pulsatility, the outflow conduit should be as short and wide as possible.

In conclusion, we report here the first acute animal study of the universal Advanced ventricular assist device intended for right, left and biventricular support (Figure 7). The pump kept the hemodynamics of calves stable with up to $4.3 \mathrm{~L} / \mathrm{min}$ as both an LVAD and an RVAD, and a regurgitant flow shut-off feature and potential pulse augmentation. The pump development is ongoing; further device assessments would include more thorough performance analysis and biocompatibility testing in a chronic model.

\section{Conflict of Interest Statement}

Fukamachi and Horvath are the device co-inventors. Horvath is a consultant for the Cleveland Clinic and may 
receive future royalties. Kuban disclosed patents on loosely related devices. Sale disclosed a financial relationship with St. Jude. All other authors reported no conflicts of interest.

The Journal policy requires editors and reviewers to disclose conflicts of interest and to decline handling or reviewing manuscripts for which they may have a conflict of interest. The editors and reviewers of this article have no conflicts of interest.

\section{References}

1. Kirklin JK, Pagani FD, Kormos RL, Stevenson LW, Blume ED, Myers SL, et al. Eighth annual INTERMACS report: special focus on framing the impact of adverse events. J Heart Lung Transplant. 2017;36:1080-6.

2. Travis AR, Giridharan GA, Pantalos GM, Dowling RD, Prabhu SD, Slaughter MS, et al. Vascular pulsatility in patients with a pulsatile- or continuous-flow ventricular assist device. J Thorac Cardiovasc Surg. 2007;133:517-24.

3. Vincent F, Rauch A, Loobuyck V, Robin E, Nix C, Vincentelli A, et al. Arterial pulsatility and circulating von Willebrand factor in patients on mechanical circulatory support. J Am Coll Cardiol. 2018;71:2106-18.

4. Witman MA, Garten RS, Gifford JR, Groot HJ, Trinity JD, Stehlik J, et al. Further peripheral vascular dysfunction in heart failure patients with a continuous-flow left ventricular assist device: the role of pulsatility. JACC Heart Fail. 2015;3: 703-11.

5. Pak SW, Uriel N, Takayama H, Cappleman S, Song R, Colombo PC, et al. Prevalence of de novo aortic insufficiency during long-term support with left ventricular assist devices. J Heart Lung Transplant. 2010;29:1172-6.

6. Bartoli CR, Zhang D, Kang J, Hennessy-Strahs S, Restle D, Howard J, et al. Clinical and in vitro evidence that subclinical hemolysis contributes to LVAD thrombosis. Ann Thorac Surg. 2018;105:807-14.

7. Starling RC, Moazami N, Silvestry SC, Ewald G, Rogers JG, Milano CA, et al. Unexpected abrupt increase in left ventricular assist device thrombosis. $N$ Engl J Med. 2014;370:33-40.

8. Sunagawa G, Byram N, Karimov JH, Horvath DJ, Moazami N, Starling RC, et al The contribution to hemodynamics even at very low pump speeds in the HVAD. Ann Thorac Surg. 2016;101:2260-4.

9. Sunagawa G, Byram N, Karimov JH, Horvath DJ, Moazami N, Starling RC, et al. In vitro hemodynamic characterization of heartmate ii at $6000 \mathrm{rpm}$ : implications for weaning and recovery. J Thorac Cardiovasc Surg. 2015;150:343-8.

10. Anderson M, Morris DL, Tang D, Batsides G, Kirtane A, Hanson I, et al. Outcomes of patients with right ventricular failure requiring short-term hemodynamic support with the Impella RP device. J Heart Lung Transplant. 2018;37: $1448-58$.

11. Takeda K, Takayama H, Colombo PC, Jorde UP, Yuzefpolskaya M, Fukuhara S, et al. Late right heart failure during support with continuous-flow left ventricular assist devices adversely affects post-transplant outcome. J Heart Lung Transplant. 2015;34:667-74.
12. Karimov JH, Sunagawa G, Horvath D, Fukamachi K, Starling RC, Moazami N. Limitations to chronic right ventricular assist device support. Ann Thorac Surg. 2016;102:651-8.

13. Horvath DJ, Karimov JH, Byram NA, Kuban BD, Sunagawa G, Moazami N, et al. Advantages of integrating pressure-regulating devices into mechanical circulatory support pumps. ASAIO J. 2019;65:e1-3.

14. Fukamachi K, Horvath DJ, Byram N, Sunagawa G, Karimov JH, Moazami N. Advanced ventricular assist device with pulse augmentation and automatic regurgitant-flow shut-off. J Heart Lung Transplant. 2016;35:1519-21.

15. Miyamoto T, Byram N, Karimov JH, Adams J, Dessoffy R, Kuban BD, et al. The design modification of advanced ventricular assist device to enhance pulse augmentation and regurgitant flow shut-off. Artif Organs. 2019;43:961-5.

16. Knierim J, Heck R, Pieri M, Schoenrath F, Soltani S, Stawowy P, et al. Outcomes from a recovery protocol for patients with continuous-flow left ventricular assist devices. J Heart Lung Transplant. 2019;38:440-8.

17. Ando M, Nishimura T, Takewa Y, Ogawa D, Yamazaki K, Kashiwa K, et al. What is the ideal off-test trial for continuous-flow ventricular-assist-device explantation? Intracircuit back-flow analysis in a mock circulation model. J Artif Organs. 2011;14:70-3.

18. Potapov EV, Schweiger M, Krabatsch T. Percutaneous balloon occlusion of a left ventricular assist device outflow cannula to facilitate evaluation of myocardial recovery. J Heart Lung Transplant. 2011;30:1300-1.

19. Fukamachi K, Shiose A, Massiello AL, Horvath DJ, Golding LA, Lee S, et al. Implantable continuous-flow right ventricular assist device: lessons learned in the development of a cleveland clinic device. Ann Thorac Surg. 2012;93: 1746-52.

20. Silva Enciso J, Tran HA, Brambatti M, Braun OO, Pretorius V, Adler ED. Management of RVAD thrombosis in biventricular HVAD supported patients: case series. ASAIO J. 2019;65:e36-41.

21. Marasco SF, Stornebrink RK, Murphy DA, Bergin PJ, Lo C, McGiffin DC. Longterm right ventricular support with a centrifugal ventricular assist device placed in the right atrium. J Card Surg. 2014;29:839-42.

22. Lo C, Gregory S, Stevens M, Murphy D, Marasco S. Banding the right ventricular assist device outflow conduit: is it really necessary with current devices? Artif Organs. 2015;39:1055-61.

23. Shah P, Ha R, Singh R, Cotts W, Adler E, Kiernan M, et al. Multicenter experience with durable biventricular assist devices. J Heart Lung Transplant. 2018;37: 1093-101.

24. Shehab S, Macdonald PS, Keogh AM, Kotlyar E, Jabbour A, Robson D, et al. Long-term biventricular Heartware ventricular assist device support—case series of right atrial and right ventricular implantation outcomes. J Heart Lung Transplant. 2016;35:466-73.

25. Ootaki Y, Saeed D, Ootaki C, Akiyama M, Fumoto H, Horai T, et al. Development of the Dexaide right ventricular assist device inflow cannula. ASAIO J. 2008;54:31-6.

Key Words: animal study, mechanical circulatory support, pulsatility, regurgitation, ventricular assist device 